\title{
Résolution des équations de Saint-Venant par la méthode des volumes finis non structurés
}

\author{
Yu-e Shi* — Kim Dan Nguyen** — The Hung Nguyen*** \\ * Research Institute for Knowledge Systems \\ P.O. Box 463 \\ 6200 AL, Maastricht \\ The Netherlands \\ yshi@riks.nl \\ ** UMR CNRS 6143 «Morphodynamique Continentale et Côtière » \\ Département de Mathématiques et Mécanique \\ Université de Caen, Campus II, Bd. Maréchal Juin \\ BP 5186, F-14032 Caen cedex \\ nguyen@meca.unicaen.fr \\ *** Institut de Mécanique, 264 Doi Can, Hanoi, Vietnam \\ nthung@im01.ac.vn
}

RÉSUMÉ. Cet article présente une résolution des équations de Saint-Venant par une méthode de projection. Un modèle numérique $2 D$ horizontal est développé par la méthode de volumes finis en maillages non structurés pour calculer des écoulements à surface libre. Deux applications sont aussi présentées dans lesquelles les bathymétries sont complexes. Ce modèle peut être appliqué au calcul des écoulements fluviaux, estuariens et côtiers.

ABSTRACT. This paper presents a resolution of the Saint-Venant equations by a projection method. Using unstructured finite-volumes methods, a 2-D horizontal shallow water numerical model is developed. Two applications with complex bathymetries are reported in this article. The model can be applied to the calculation of river, estuarine and coastal flows.

MOTS-CLÉS : équations de Saint-Venant, modélisation numérique, volumes finis non structurés, technique de projection.

KEYWORDS: Saint-Venant equations, numerical models, unstructured finite-volumes ; projection technique.

DOI:10.3166/REMN.16.723-747 C 2007 Lavoisier, Paris 


\section{Introduction}

La méthode des volumes finis consiste à discrétiser le domaine de l'écoulement en une multitude de volumes de contrôle, puis à effectuer des bilans de masse et de quantité de mouvement sur ces petits volumes. L'intérêt de la méthode des volumes finis réside dans le fait qu'elle assure la conservation de la masse, propriété importante à respecter par tous les calculs des écoulements de fluides, et permet de réduire un ordre de dérivées des équations aux dérivées partielles. En maillage non structuré, la méthode des volumes finis nous offre, par ailleurs, une prise en compte parfaite de la géométrie complexe du domaine de calcul.

Dans les modèles de volumes finis existants, qui sont basés sur la résolution des équations de Saint-Venant, deux techniques ont été principalement utilisées : le solveur de l'approximation Riemann de Roe (RARS : Roe's Approximate Riemann Solver) et la méthode de projection.

Le RARS a été initialement proposé pour la résolution de l'équation hyperbolique homogène (Roe, 1981). En 1989, (Fennema et Chaudhry, 1989) sont parmi les premiers qui utilisent un RARS pour résoudre les équations de SaintVenant en formulation conservative avec un schéma de McCormark et de Gabutti. Le RARS est ensuite développé avec un schéma TVD (Total Variation Diminishing) pour les problèmes de rupture de barrage unidimensionnels par (Alcrudo et GarciaNavarro, 1993) et (Garcia-Navarro et al., 1992) et bidimensionnels par (Hanich, 1996). Les MVF utilisées dans ces travaux restent en maillage structuré. Bien que le RARS puisse correctement simuler les écoulements discontinus et les chocs, des oscillations indésirables puissent cependant se produire lorsque les termes source sont importants.

Le RARS en MVF est étendu dans (Sleigh et al., 1998; Chan et Anastasiou, 1999) à des maillages non structurés. Sleigh et al., (1998) ont introduit un limiteur du type (Van Leer, 1977) dans leur schéma «Upwind» pour traiter les termes d'advection. Chan et Anastasiou, (1999) ont développé un schéma «Upwind » du $2^{\text {nd }}$ ordre du type Godunov. Cependant, le traitement des termes source causé notamment par l'irrégularité du fond n'a pas encore pris une place appropriée dans ces travaux.

En 2001, dans leur modèle utilisant un RARS en volumes finis non structurés, (Zhou et al., 2001) commencent à prêter leur attention au traitement des termes source. (Brufau et al., 2002) ont présenté un traitement spécial qui décompose les termes source en deux termes «inward» et «outward». Restant fidèles au RARS en volumes finis non structurés, (Brufau et al., 2002, 2004) ont proposé des techniques numériques pour traiter le problème lié aux terrains découvrant et recouvrant en respectant la conservation de la masse d'eau. (Murillo et al., 2005) ont proposé l'extension du RARS développé par (Brufau et al., 2002; 2004) à des grands pas de temps de calcul qui peuvent aller jusqu'à une valeur du nombre de CFL de 4. Dans les articles de (Calleffi et al., 2006 ; Harten, 1987; Vukovic et Sopta, 2002), les termes source sont calculés par la reconstruction essentiellement non oscillatoire (ENO - Essentially Non-Oscillatory) et pondérée essentiellement 
non oscillatoire (WENO - Weighted Essentially Non-Oscillatory). Cependant, les méthodes développées pour traiter les termes source restent encore très complexes.

La méthode de projection (MP) proposée indépendamment par (Chorin, 1968 ; 1969 ; Temam, 1968 ; 1977) est la technique fréquemment utilisée pour la résolution numérique des équations de Navier-Stokes en variables primitives. Cette méthode, est basée sur l'utilisation d'un champ de vecteurs vitesses auxiliaires (en ôtant les termes de pression des équations de Navier-Stokes), et sur l'aboutissement à une équation de Poisson pour la pression (comme la seule inconnue) par un couplage entre l'équation de continuité et celle de quantité de mouvement. Une fois la pression obtenue, le champ des vecteurs vitesses à divergence nulle est déterminé par la correction du champ de vitesses auxiliaire avec la prise en compte, cette fois, des termes de pression. Stevens et al., (2002) ont précisé que le terme «Méthode de projection » est à l'origine du fait qu'un champ de vitesse peut être décomposé en un champ non divergent et un champ de gradient d'une grandeur scalaire. Les méthodes qui déterminent cette grandeur scalaire par la résolution d'un problème de Poisson et par la soustraction du gradient de cette grandeur du champ de vitesse original, s'appellent «méthode de projection». (Guillou, 1996; Guillou et Nguyen, 1999) ont utilisé la MP pour la résolution des équations de Saint-Venant dans un maillage curviligne et donc structuré. Ils ont montré que l'utilisation d'un maillage non décalé pour la résolution des équations de Saint-Venant peut produire des oscillations indésirables non seulement dans les champs de la surface libre, mais également dans ceux de vitesses. La MP se montre très efficace pour calculer les écoulements à surface libre sur des fonds très irréguliers et nécessite aucun traitement spécial pour des termes source. A présent, il n'existe cependant aucun travail abordant la résolution des équations de Saint-Venant par une MP en volumes finis non structurés, permettant une modélisation parfaite des domaines de calcul en géométrie arbitraire.

Cet article présente un modèle numérique $2 \mathrm{D}$ qui est basé sur la résolution des équations de Saint-Venant par la MP en utilisant une méthode des volumes finis en maillages non structurés. Le choix de la MP a été motivé en prévision des applications de ce modèle à des problèmes qui représentent des termes sources importants dus principalement à une variation rapide et à une forte irrégularité du fond. L'interpolation pondérée de la pression proposée par (Rhie et al., 1983) est utilisée afin d'éviter les oscillations numériques provoquées par l'utilisation de la méthode de projection dans un maillage non décalé. La validation du modèle par deux cas-tests et l'application dans l'étude du barrage de Malpasset ainsi que dans l'étude de la rupture des digues du district Thanh-Ha (Nord du Vietnam) sont également présentées dans cet article. 


\section{Modélisation des écoulements bidimensionnels horizontaux}

\subsection{Equations de Saint-Venant}

Les équations dites « de Barré de Saint-Venant», publiées en 1871 (Saint-Venant, 1871), occupent encore aujourd'hui une place primordiale dans les études en hydraulique maritime ou fluviale. Ces équations décrivent les écoulements à surface libre en eaux peu profondes, d'où leur appellation anglaise «Shallow Water Equations ». Elles sont obtenues à partir d'une intégration verticale des équations 3D de Navier-Stokes en utilisant l'hypothèse de pression hydrostatique et l'approximation de Boussinesq. Les lecteurs peuvent trouver les détails de la dérivation des équations de Saint-Venant dans les littératures (Herouvet, 2003 ; Tan, 1992 ; Ryhming, 1985). Les équations de Saint-Venant se composent de l'équation de continuité et celle de quantité du mouvement dans les directions horizontales $x$ et $y$.

Equation de continuité

$$
\frac{\partial Z_{s}}{\partial t}+\frac{\partial(h u)}{\partial x}+\frac{\partial(h v)}{\partial y}=0
$$

Equations de quantité de mouvement

$$
\begin{aligned}
& \frac{\partial(h u)}{\partial t}+\frac{\partial\left(h u^{2}\right)}{\partial x}+\frac{\partial(h u v)}{\partial y}=\frac{\partial}{\partial x}\left[A_{H} \frac{\partial(h u)}{\partial x}\right]+\frac{\partial}{\partial y}\left[A_{H} \frac{\partial(h u)}{\partial y}\right]-g h \frac{\partial Z_{s}}{\partial x}-\frac{\tau_{b x}}{\rho_{o}}+f(h v) \\
& \frac{\partial(h v)}{\partial t}+\frac{\partial(h u v)}{\partial x}+\frac{\partial\left(h v^{2}\right)}{\partial y}=\frac{\partial}{\partial x}\left[A_{H} \frac{\partial(h v)}{\partial x}\right]+\frac{\partial}{\partial y}\left[A_{H} \frac{\partial(h v)}{\partial y}\right]-g h \frac{\partial Z_{s}}{\partial y}-\frac{\tau_{b y}}{\rho_{o}}-f(h u)
\end{aligned}
$$

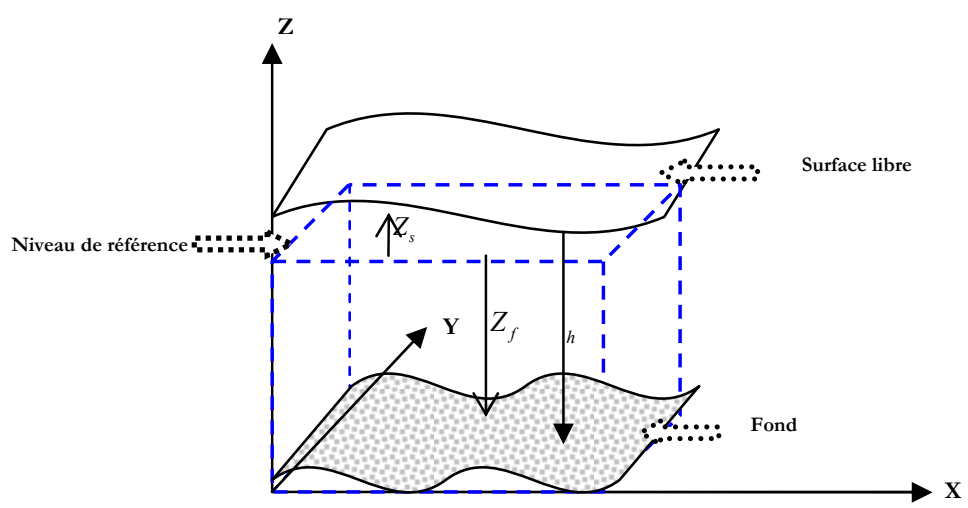

Figure 1. Notations des variables 
La figure 1 présente les repères de travail et les notations des variables physiques. Dans les équations [1] à [3], $A_{H}$ est le coefficient de dispersion horizontale, $g$ est l'accélération gravitationnelle, $f$ est le paramètre de Coriolis, $u$ et $v$ sont les composantes de la vitesse dans les directions $x$ et $y$, respectivement, $h$ est la profondeur totale de l'écoulement, $Z_{s}$ est la dénivellation à surface libre, $\rho_{o}$ est la masse volumique de référence de l'eau, $\tau_{b x}$ et $\tau_{b y}$ sont les contraintes de cisaillement dans les directions $x$ et $y$, déterminées par $\left(\tau_{b x}, \tau_{b y}\right)=\rho_{0} \frac{g}{C_{h}^{2}} \sqrt{u^{2}+v^{2}}(u, v)$ où $C_{h}$ est le coefficient de Chézy.

Si l'on ôte les termes source (trois derniers termes : gradients de la surface libre, contraintes de frottement et force de Coriolis) du second membre des équations [2] et [3], ces équations prendront la forme d'une équation de transport. En conséquence, la présentation des techniques numériques pour la résolution des équations de SaintVenant passe d'abord à celle de l'équation de transport d'une variable scalaire quelconque.

\subsection{Résolution de l'équation de transport par une MVF non structurée}

Dans cette section, nous donnons une description de la discrétisation de l'équation de transport telle qu'elle a été proposée dans l'article de (Lien, 2000), avant de l'appliquer aux équations de Saint-Venant. Considérons l'équation de transport suivante :

$$
\int_{\Omega} \frac{\partial \phi}{\partial t} d \Omega+\int_{\Omega}\left(\frac{\partial(u \phi)}{\partial x}+\frac{\partial(v \phi)}{\partial y}\right) d \Omega=\int_{\Omega}\left(\frac{\partial}{\partial x}\left(\Gamma \frac{\partial \phi}{\partial x}\right)+\frac{\partial}{\partial y}\left(\Gamma \frac{\partial \phi}{\partial y}\right)\right) d \Omega
$$

où, $\phi=\phi(x, y, t)$ est une valeur scalaire ; $u$ et $v$ sont les composantes du vecteur vitesse $\vec{U}$; et $\Gamma$ est le coefficient de diffusion.

\subsubsection{Approximation des termes de convection}

L'application du théorème de Green, pour une fonction quelconque $\phi$ au moins une fois dérivable, donne [5].

$$
\frac{\partial \phi}{\partial x}=\frac{1}{\Omega} \int \phi d y, \frac{\partial \phi}{\partial y}=-\frac{1}{\Omega} \int \phi d x
$$



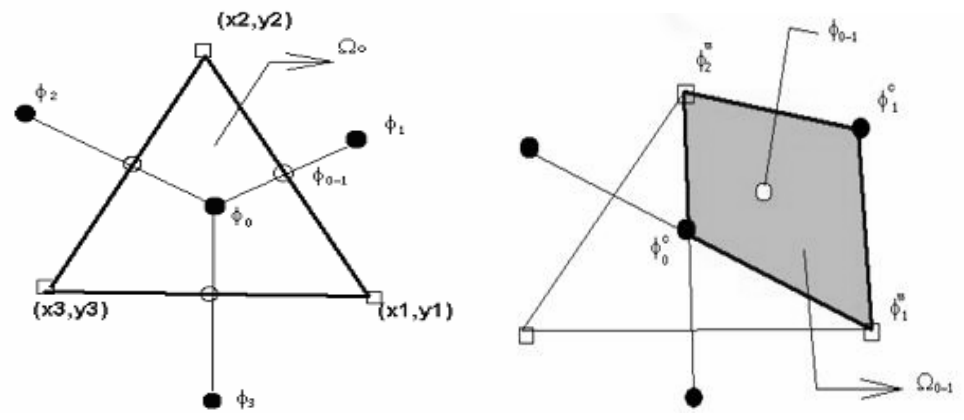

Figure 2. Volumes de contrôle pour les flux convectifs traversant les faces (gauche) et pour le flux diffusif traversant la face (1-2) (droit)

L'intégration est effectuée sur le domaine de calcul $\Omega$ de frontière $\partial \Omega$. On suppose que le domaine $\Omega$ est discrétisé par $\mathrm{N}$ volumes de contrôle. Considérons le volume de contrôle $\Omega_{o}$ (voir la figure 2). Les termes de convection de l'équation [4] peuvent être calculés par la relation :

$$
\int_{\Omega_{0}}\left(\frac{\partial(u \phi)}{\partial x}+\frac{\partial(\nu \phi)}{\partial y}\right) d \Omega=\int_{\Omega_{0}} \vec{\nabla} \cdot(\vec{U} \phi) d \Omega=\int_{\partial \Omega_{0}}(\vec{U} \phi) \cdot \vec{n} d \gamma=\sum_{m} C_{0-m} \phi_{0-m}
$$

où $\vec{U}$ est le vecteur vitesse ; $\vec{n}$ est le vecteur normal à la frontière ; $d \gamma$ est un élément de la frontière $\partial \Omega_{o} ; C_{0-m}$ est le flux de la masse d'eau traversant la facette $m$ ( $m=1,2$ et 3$) ; \phi_{0-m}$ est la valeur de $\phi$ sur la facette $m$. $C_{0-m}$ peut être déterminé par :

$$
\begin{aligned}
& C_{0-1}=u_{0-1} \Delta y_{12}-v_{0-1} \Delta x_{12} \\
& C_{0-2}=u_{0-2} \Delta y_{23}-v_{0-2} \Delta x_{23} \\
& C_{0-3}=u_{0-3} \Delta y_{31}-v_{0-3} \Delta x_{31}
\end{aligned}
$$

où $u_{0-m}$ et $v_{0-m}(\mathrm{~m}=1,2$ et 3$)$ sont les composantes du vecteur vitesse sur la facette $m ; \Delta x_{m, n}$ et $\Delta y_{m, n}$ sont la projection de la facette $m$ dans la direction $x$ et $y$, respectivement. Afin d'éviter les oscillations numériques provoquées par l'utilisation de la méthode de projection dans un maillage non décalé, les composantes $u_{0-m}$ et $v_{0-m}$ sont évaluées par l'interpolation des vitesses (Guillou et Nguyen, 1999) ou par l'interpolation pondérée de la pression (Rhie et Chow, 1983).

La valeur de $\phi$ sur les facettes est calculée grâce au schéma «upwind» du deuxième ordre, comme suit : 


$$
\phi_{0-m}=\left\{\begin{array}{lll}
\phi_{0}+(\nabla \phi)_{0} \cdot \frac{\Delta \vec{r}_{0-m}}{2} & \text { si } & C_{0-m}>0 \\
\phi_{m}-(\nabla \phi)_{m} \cdot \frac{\Delta \vec{r}_{0-m}}{2} & \text { si } & C_{0-m}<0
\end{array} \text { où } \quad \Delta \vec{r}=(\Delta x, \Delta y)\right.
$$

où le gradient de $\phi$ peut être déterminé par un schéma «Upwind » aux moindres carrés ( Kobayashi et al., 1999).

\subsubsection{Approximation des termes de diffusion}

L'application de [5] au volume de contrôle pour le flux diffusif traversant le côté donne 1-2 :

$$
\begin{aligned}
& \left(\frac{\partial \phi}{\partial x}\right)_{0-1}=\frac{1}{2 \Omega_{0-1}}\left[\left(\phi_{1}^{c}-\phi_{0}^{c}\right) \Delta y_{12}-\left(\phi_{2}^{v}-\phi_{1}^{v}\right) \Delta y_{01}\right] \\
& \left(\frac{\partial \phi}{\partial y}\right)_{0-1}=\frac{-1}{2 \Omega_{0-1}}\left[\left(\phi_{1}^{c}-\phi_{0}^{c}\right) \Delta x_{12}-\left(\phi_{2}^{v}-\phi_{1}^{v}\right) \Delta x_{01}\right]
\end{aligned}
$$

La valeur du $\phi^{v}$ est obtenue en faisant la moyenne de toutes les valeurs au centre des cellules voisines :

$$
\phi_{0}^{v}=\frac{\sum \phi_{i}^{c}\left(1 / L_{i}\right)}{\sum\left(1 / L_{i}\right)}
$$

où $l_{i}$ est la distance entre le sommet et le centre de volume de contrôle $l_{i}$ (voir la figure 3)

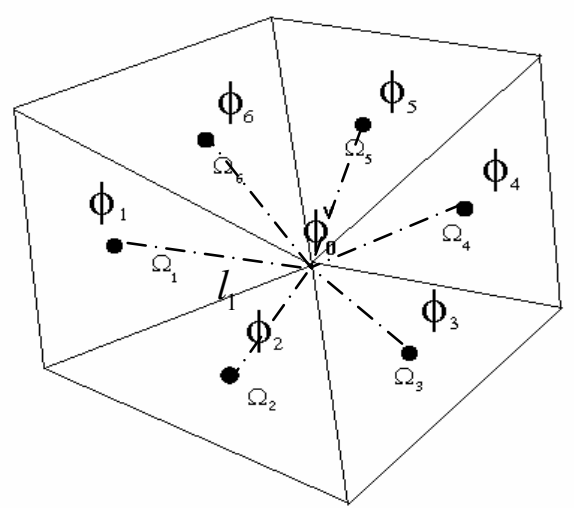

Figure 3. Interpolation de la valeur ae paux sommets 
Le résultat final de l'intégration des termes de diffusion est :

$$
\begin{aligned}
& \int_{\Omega}\left[\frac{\partial}{\partial x}\left(\Gamma \phi_{x}\right)+\frac{\partial}{\partial y}\left(\Gamma \phi_{y}\right)\right] d \Omega \\
& =\frac{\Gamma_{0-1}}{2 \Omega_{0-1}}\left[\left(\phi_{1}-\phi_{0}\right)\left(\Delta x_{12}^{2}+\Delta y_{12}^{2}\right)-\left(\phi_{2}^{v}-\phi_{1}^{v}\right)\left(\Delta x_{01} \Delta x_{12}+\Delta y_{01} \Delta y_{12}\right)\right] \\
& +\frac{\Gamma_{0-2}}{2 \Omega_{0-2}}\left[\left(\phi_{2}-\phi_{0}\right)\left(\Delta x_{23}^{2}+\Delta y_{23}^{2}\right)-\left(\phi_{3}^{v}-\phi_{2}^{v}\right)\left(\Delta x_{02} \Delta x_{23}+\Delta y_{02} \Delta y_{23}\right)\right] \\
& +\frac{\Gamma_{0-3}}{2 \Omega_{0-3}}\left[\left(\phi_{3}-\phi_{0}\right)\left(\Delta x_{31}^{2}+\Delta y_{31}^{2}\right)-\left(\phi_{1}^{v}-\phi_{3}^{v}\right)\left(\Delta x_{03} \Delta x_{31}+\Delta y_{03} \Delta y_{31}\right)\right]
\end{aligned}
$$

\subsubsection{Système des équations linéaires}

En remplaçant des termes de [4] par [6] et [11], on obtient [12] pour chaque volume de contrôle. Le terme source $S_{\phi}$ est déterminé par [13].

$$
\begin{aligned}
A_{0} \phi_{0}^{n+1}= & \sum_{m}^{3} A_{m} \phi_{m}^{n+1}+S_{\phi} \\
S_{\phi}=\phi_{0}^{n} & +\frac{d t}{\Omega_{0}}\left\{-\frac{\Gamma_{0-1}}{2 \Omega_{0-1}}\left[\left(\phi_{2}^{v}-\phi_{1}^{v}\right)\left(\Delta x_{01} \Delta x_{12}+\Delta y_{01} \Delta y_{12}\right)\right]\right\} \\
& +\frac{d t}{\Omega_{0}}\left\{-\frac{\Gamma_{0-2}}{2 \Omega_{0-2}}\left[\left(\phi_{3}^{v}-\phi_{2}^{v}\right)\left(\Delta x_{02} \Delta x_{23}+\Delta y_{02} \Delta y_{23}\right)\right]\right\} \\
& +\frac{d t}{\Omega_{0}}\left\{-\frac{\Gamma_{0-3}}{2 \Omega_{0-3}}\left[\left(\phi_{1}^{v}-\phi_{3}^{v}\right)\left(\Delta x_{03} \Delta x_{31}+\Delta y_{03} \Delta y_{31}\right)\right]\right\} \\
& +\frac{d t}{\Omega_{0}}\left\{-\frac{1}{2} \sum_{m}^{3}\left[C_{0-m}^{+}(\nabla \phi)_{0} \cdot \Delta \vec{r}_{0-m}-C_{0-m}^{-}(\nabla \phi)_{m} . \Delta \vec{r}_{0-m}\right]\right\}
\end{aligned}
$$

[12] permet d'établir un système d'équations linéaires pour $N$ volumes de contrôle. Ce système peut être résolu implicitement par une technique de SOR (Successive Over Relaxation, Tannehill et al., 1997).

\subsection{Résolution des équations de Saint-Venant par une méthode de projection}

La méthode de projection de (Chorin, 1968), a été appliquée ici pour résoudre les équations de Saint-Venant (Guillou et Nguyen, 1999), après les étapes successives de convection - diffusion, de propagation de l'onde de surface et de correction de vitesses. L'étape de convection-diffusion, établie en ôtant les termes source des équations [2] et [3], fournira un champ de vitesse auxiliaire dont sa divergence est non nulle. L'étape de propagation consiste à combiner les équations [1]-[3] afin d'aboutir à une équation de Poisson pour la dénivellation de la surface libre, seule inconnue à déterminer. Le champ de vitesse auxiliaire est finalement corrigé dans l'étape de correction, une fois les nouvelles valeurs de dénivellation de la surface libre déterminées. 


\subsubsection{Etape de convection-diffusion}

En appliquant la technique présentée dans la section 2.2 aux équations de transport [4], nous obtenons un système d'équation de la forme de [12] avec $q_{x}{ }^{*}$ et $q_{y}{ }^{*}$ comme les intermédiaires inconnus à déterminer.

$$
\begin{aligned}
& \frac{\partial q_{x}^{*}}{\partial t}+\frac{\partial\left(u q_{x}^{*}\right)}{\partial x}+\frac{\partial\left(v q_{x}^{*}\right)}{\partial y}=A_{H}\left[\frac{\partial^{2} q_{x}^{*}}{\partial x^{2}}+\frac{\partial^{2} q_{x}^{*}}{\partial y^{2}}\right] \\
& \frac{\partial q_{y}^{*}}{\partial t}+\frac{\partial\left(u q_{y}^{*}\right)}{\partial x}+\frac{\partial\left(v q_{y}^{*}\right)}{\partial y}=A_{H}\left[\frac{\partial^{2} q_{y}^{*}}{\partial x^{2}}+\frac{\partial^{2} q_{y}^{*}}{\partial y^{2}}\right]
\end{aligned}
$$

où $\left(q_{x}, q_{y}\right)=(h u, h v)$ sont les composantes du vecteur débit unitaire.

\subsubsection{Etape de propagation de l'onde}

L'élimination de $h u$ et $h v$ dans [2] et [3] grâce à l'utilisation de [1], donne :

$$
\begin{aligned}
& {\left[1-\frac{\gamma^{2} g h}{A}\left(\frac{\partial^{2}}{\partial x^{2}}+\frac{\partial^{2}}{\partial y^{2}}\right)\right] \delta Z_{s}=\frac{B}{A} \quad \delta Z_{s}=Z_{s}^{n+1}-Z_{s}^{n}} \\
& \left\{\begin{array}{l}
B=\gamma g h\left(\frac{\partial^{2} Z_{s}^{n}}{\partial x^{2}}+\frac{\partial^{2} Z_{s}^{n}}{\partial y^{2}}\right)-\frac{\gamma}{d t}\left(\frac{\partial q_{x}}{\partial x}+\frac{\partial q_{y}}{\partial y}\right)-\gamma\left(\frac{\partial L_{x}}{\partial x}+\frac{\partial L_{y}}{\partial y}\right)-\frac{1-\gamma}{d t}\left(\frac{\partial q_{x}^{n}}{\partial x}+\frac{\partial q_{y}^{n}}{\partial y}\right) \\
A=d t^{-1}\left(d t^{-1}+\gamma F\right) \\
F=\frac{g}{C_{h}^{2} h^{2}} \sqrt{\left(q_{x}^{n}\right)^{2}+\left(q_{y}^{n}\right)^{2}} \\
L_{x}=f q_{y}^{n} \\
L_{y}=-f q_{x}^{n}
\end{array}\right.
\end{aligned}
$$

L'application de [6] et [11] à [15] donne un système d'équations linéaire comme suit :

$$
A_{Z 0} \delta Z_{s 0}=\sum_{m}^{3} A_{Z m} \delta Z_{s m}+S_{Z}
$$




$$
\begin{aligned}
& A_{Z 1}=\frac{D_{0-1}\left(\Delta x_{12}^{2}+\Delta y_{12}^{2}\right)}{2 \Omega_{0-1}} \\
& A_{Z 2}=\frac{D_{0-2}\left(\Delta x_{23}^{2}+\Delta y_{23}^{2}\right)}{2 \Omega_{0-2}} \\
& A_{Z 3}=\frac{D_{0-3}\left(\Delta x_{31}^{2}+\Delta y_{31}^{2}\right)}{2 \Omega_{0-3}} \\
& A_{Z 0}=\Omega_{0}+\left(A_{1}+A_{2}+A_{3}\right)
\end{aligned}
$$

L'équation [17] peut être résolue par une technique de SOR implicite. Le terme source $S_{Z}$ peut être déduit par la comparaison de [15] et [17] en développant respectivement chacune d'elles avec [16] et [18]. Ainsi, on peut obtenir la dénivellation à surface libre au pas de temps suivant $Z_{s}^{n+1}$.

\subsubsection{Etape de correction de vitesse}

Après l'obtention de $Z_{s}^{n+1}$, les débits unitaires peuvent être corrigés comme suit :

$$
\begin{aligned}
& q_{x}^{n+1}=\frac{q_{x}^{*}-\gamma g h d t \frac{\partial Z_{s}^{n+1}}{\partial x}-(1-\gamma) g h d t \frac{\partial Z_{s}^{n}}{\partial x}+L_{x} d t-(1-\gamma) F q_{x}^{n} d t}{1+\gamma F d t} \\
& q_{y}^{n+1}=\frac{q_{y}^{*}-\gamma g h d t \frac{\partial Z_{s}^{n+1}}{\partial y}-(1-\gamma) g h d t \frac{\partial Z_{s}^{n}}{\partial y}+L_{y} d t-(1-\gamma) F q_{y}^{n} d t}{1+\gamma F d t}
\end{aligned}
$$

En utilisant la discrétisation de [19] et [20] par [9] on peut obtenir les vitesses corrigées.

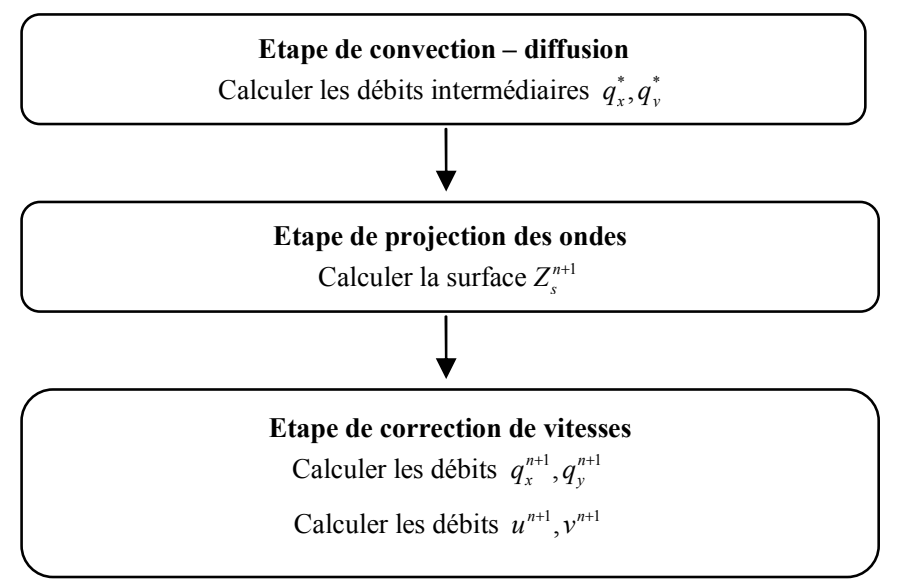

Figure 4. Organigramme de calcul 
L'organigramme de calcul suivant ces trois étapes est présenté dans la figure 4. Comme les systèmes d'équations linéaires dans les étapes de convection-diffusion (système [12]) et de propagation des ondes (système [17]) sont résolus par une technique implicite du type SOR, les résultats numériques pourraient être théoriquement stables même avec un pas de calcul assez large. Nos expériences numériques montrent cependant que pour des cas d'applications réelles sur des fonds très irréguliers, le nombre de Courant doit rester inférieur à 1 pour obtenir des solutions stables.

\section{Validations}

\subsection{Rupture partielle d'un barrage de réservoir}

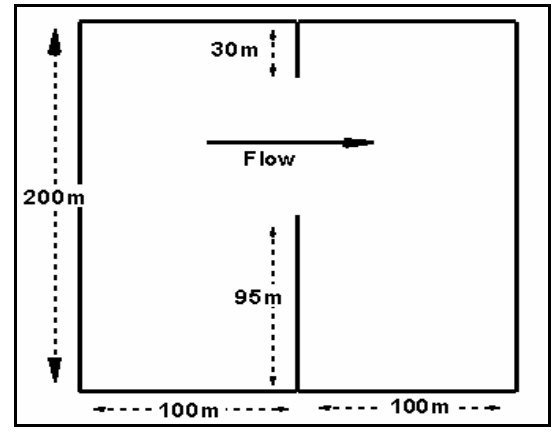

Figure 5. Géométrie de la rupture partielle du barrage

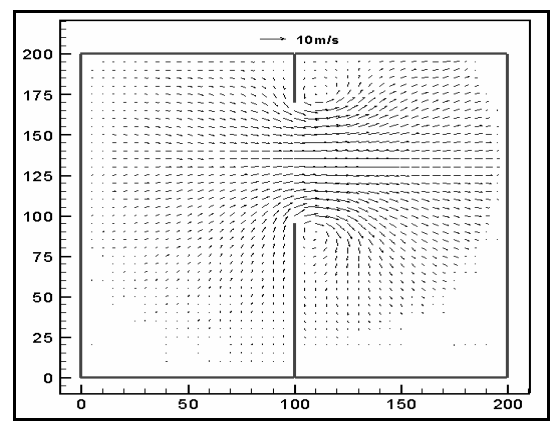

Figure 6. Champ de vitesse à $t=10.2 \mathrm{~s}$

L'objectif de ce cas-test est de vérifier la capacité de capture des ondes de choc bidimensionnelles par le modèle. Il s'agit d'un réservoir fermé de section carrée de $200 \mathrm{~m}$. Initialement, les profondeurs d'eau sont de $10 \mathrm{~m}$ en amont et de $5 \mathrm{~m}$ en aval 
du barrage. A l'instant $\mathrm{t}=0$, le barrage du réservoir est simultanément en rupture partielle sur une longueur de $75 \mathrm{~m}$ (voir la figure 5). Ce problème a été étudié par (Fennema et al., 1990). Le domaine a été discrétisé en 3200 triangles. La simulation dure $10.2 \mathrm{~s}$. Le pas de temps est de $0.01 \mathrm{~s}$.

La figure 6 montre le champ de vitesse à l'instant $t=10.2 \mathrm{~s}$. La figure 7 et la figure 8 représentent les contours de la surface libre en deux et en trois dimensions à $t=7.2 \mathrm{~s}$ après la rupture. Ces résultats semblent très similaires à ceux présentés par les autres modèles (Fennema et al., 1990 ; Zhao et al.,1994 ; Hanich, 1996). Bien que le modèle actuel soit basé sur une méthode de projection, il peut cependant simuler des écoulements hyperboliques même en présence de la discontinuité des surfaces de l'eau et de vitesses.

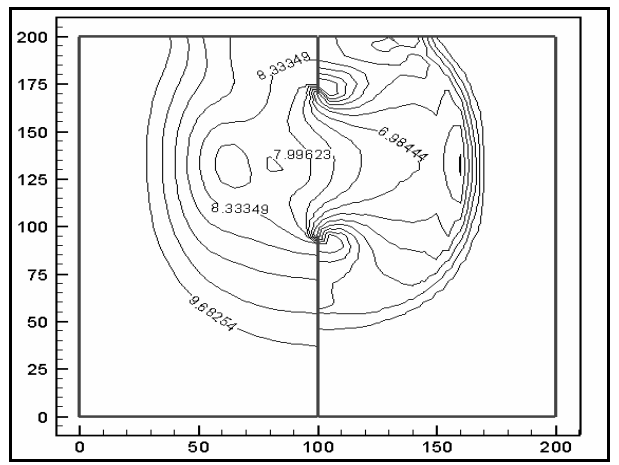

Figure 7. Elévation de la surface de l'eau à $t=7.2 \mathrm{~s}$ en $2 \mathrm{D}$

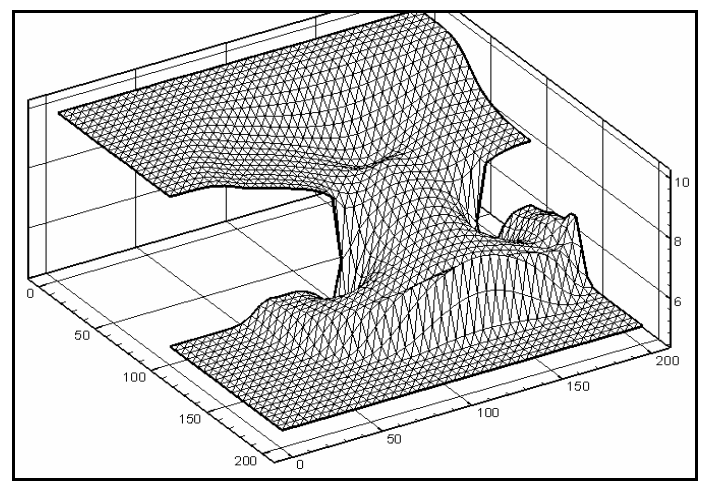

Figure 8. Elévation de la surface de l'eau à $t=7.2 \mathrm{~s}$ en $3 D$ 


\subsection{Ecoulement forcé par jet dans un réservoir circulaire}

Un écoulement régulier forcé par jet à un faible nombre de Reynolds est étudié en eau peu profonde afin d'examiner les capacités du modèle à calculer des écoulements horizontaux dans une géométrie complexe. Le réservoir a un rayon de $R=0.75 \mathrm{~m}$. On place deux canaux de longueur $L=0.3 \mathrm{~m}$ et de largeur $b=0.157 \mathrm{~m}$ sur l'entrée et la sortie. La profondeur de l'eau est uniforme $h_{0}=0.1 \mathrm{~m}$. La vitesse initiale est $U_{0}=0.1 \mathrm{~m} / \mathrm{s}$. On impose, à l'entrée le profil parabolique de vitesses suivant :

$$
U=1.5 U_{I}\left(1-\frac{y^{2}}{(b / 2)^{2}}\right)
$$

L'élévation de l'eau est nulle et reste constante à la sortie. Le nombre de Reynolds est $R e=10$ (le coefficient de dispersion horizontale étant $A_{H}=0.00078$ ), défini selon $\operatorname{Re}=U_{I} b / 2 A_{H}$ où $U_{I}=0.1 \mathrm{~m} / \mathrm{s}$ es la vitesse moyenne d'entrée.

Une grille de 1800 éléments triangulaires (figure 9), équivalente à une maille de dimension 31 x 31 de B\&K (Borthwick et Karr, 1993), est employée. Le pas de temps est de $0.035 \mathrm{~s}$. L'état d'équilibre est atteint après $80 \mathrm{~s}$. La figure 10 présente l'élévation de la surface libre d'eau à $\mathrm{t}=80 \mathrm{~s}$. Les résultats calculés sont comparés à la solution numérique de B\&K. La figure 11 montre le champ de vitesses mettant en évidence l'extension du jet lors de son entrée, puis sa contraction lors de sa sortie du réservoir comme dans les résultats obtenus par B\&K. On peut y voir également les deux zones symétriques de recirculation, établies de chaque côté du jet. La figure 12 montre le profil de la composante de vitesse $U$ sur le milieu du réservoir. Une excellente concordance avec les résultats de B\&K y est remarquée.

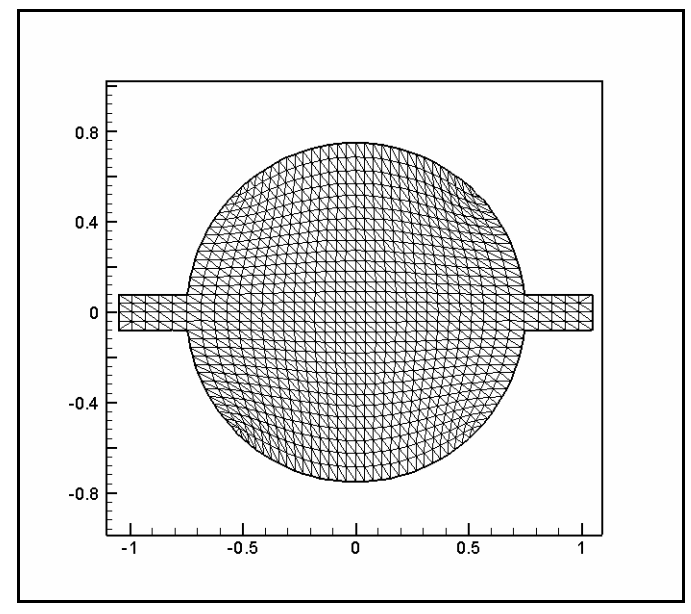

Figure 9. Maillage 1800 éléments 


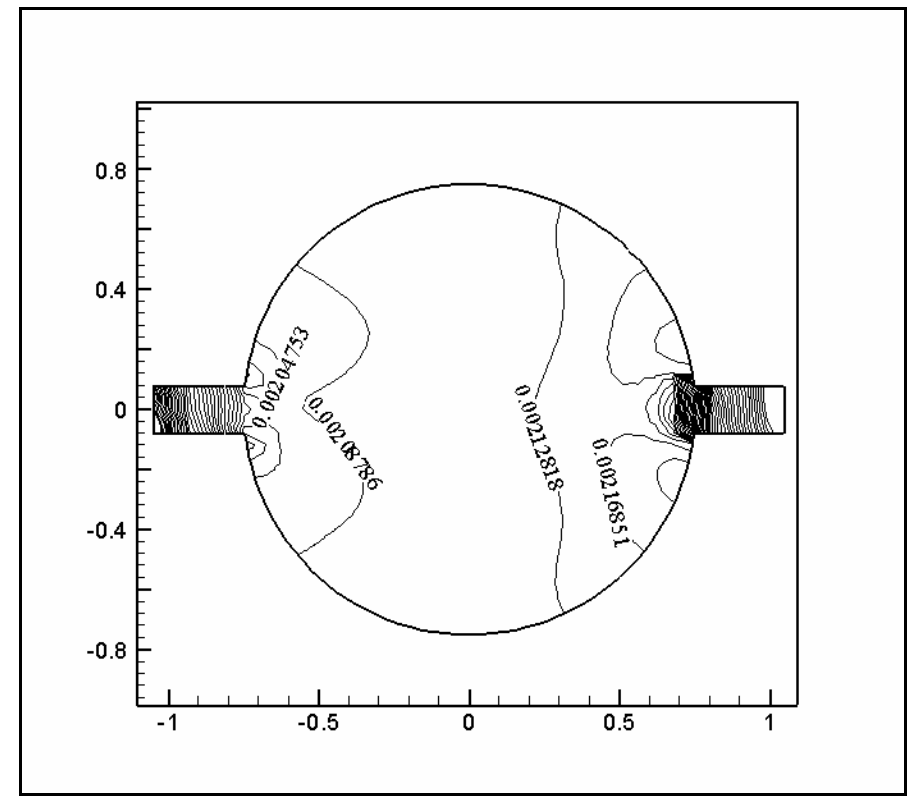

Figure 10. Elévation de la surface d'eau à $t=80 \mathrm{~s}$

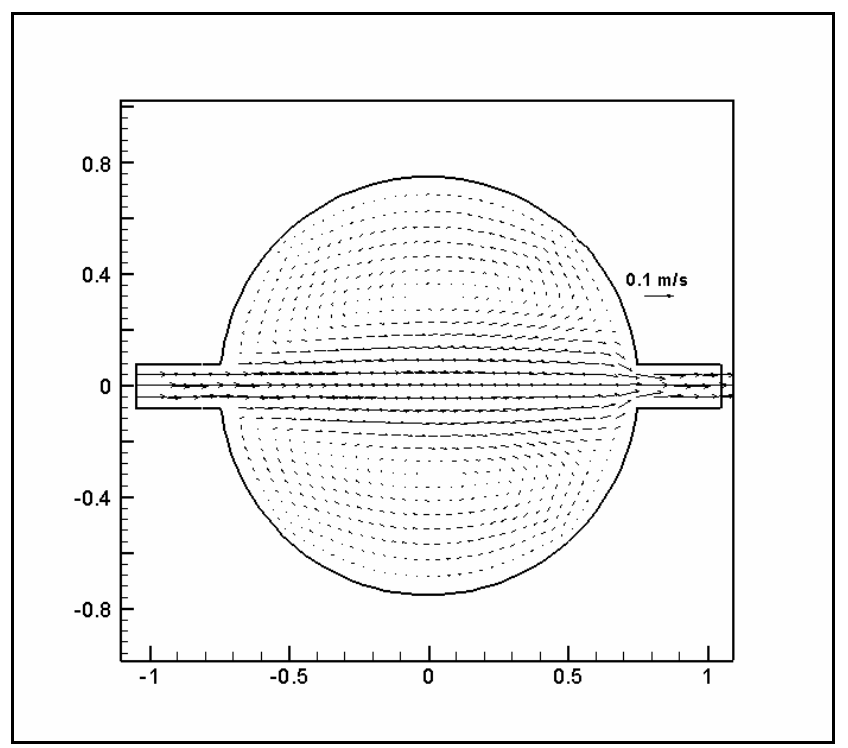

Figure 11. Champ de vitesse à $t=80 \mathrm{~s}$ 


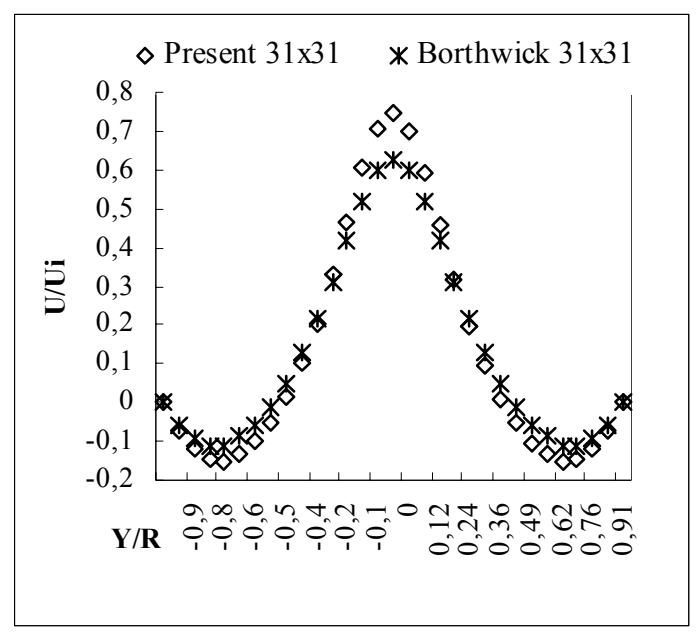

Figure 12. Profil de vitesses $U$ sur la ligne moyenne $(y=0)$

\section{Applications}

\subsection{Onde de rupture du barrage de Malpasset}

Le barrage de Malpasset a été construit pour l'irrigation et pour la réserve d'eau potable. Il était situé dans un rétrécissement de la vallée du Reyran (Département du Var, France), à 12 kilomètres de Fréjus sur la Côte d'Azur (Herouvet, 2003). L'accident de Malpasset en 1959 est un rare exemple de rupture totale et instantanée d'un barrage voûte.

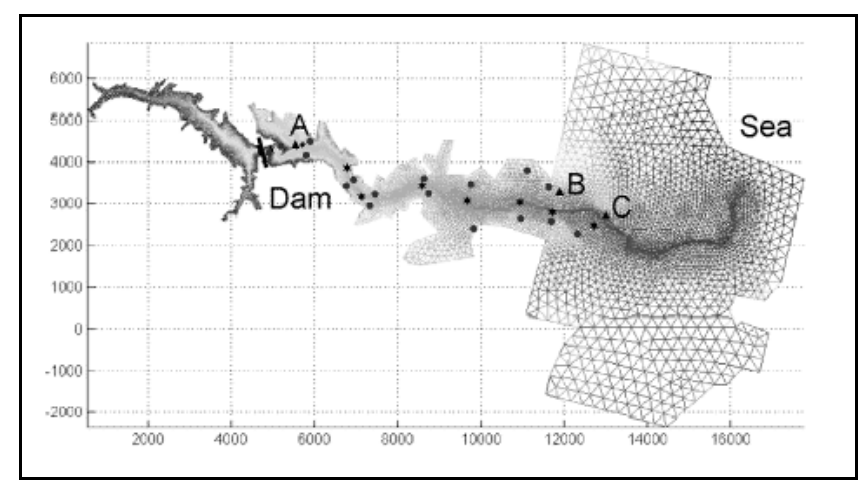

Figure 13. Contour du domaine de calcul 


\begin{tabular}{|c|c|c|c|c|c|c|c|c|}
\hline & \multicolumn{2}{|c|}{ Coefficient } & \multicolumn{5}{c|}{ Temps d'arrivée de crues (s) } \\
\cline { 2 - 9 } & Frottement & $A_{H}\left(\mathrm{~m}^{2} / \mathrm{s}\right)$ & \multicolumn{2}{|c|}{ A } & \multicolumn{2}{c|}{ B } & \multicolumn{2}{c|}{ C } \\
\hline Observations & & & 100 & Erreur & 1240 & Erreur & 1420 & Erreur \\
\hline $\begin{array}{c}\text { Valiani } \text { et al. } \\
(1996)\end{array}$ & $n=0.033 \mathrm{~s} / \mathrm{m}^{1 / 3}$ & & 98 & $-2 \%$ & 1305 & $5 \%$ & 1401 & $-1 \%$ \\
\hline $\begin{array}{c}\text { TELEMAC } \\
\text { (Hervouet, } \\
\text { 2001) }\end{array}$ & $K_{s}=30 \mathrm{~m}^{1 / 3} / \mathrm{s}$ & 1 & 111 & $11 \%$ & 1287 & $4 \%$ & 1436 & $1 \%$ \\
\hline Notre modèle & $C_{h}=25 \mathrm{~m}^{1 / 2} / \mathrm{s}$ & 15 & $\mathbf{8 5}$ & $\mathbf{- 1 5 \%}$ & $\mathbf{1 2 3 0}$ & $\mathbf{- 1 \%}$ & $\mathbf{1 3 9 6}$ & $\mathbf{- 2 \%}$ \\
\hline
\end{tabular}

Tableau 1. Temps d'arrivée de crues aux transformateurs $A, B$ et $C$

La simulation est proposée avec les conditions suivantes, qui sont identiques au modèle physique. Les dimensions d'étude sont $17500 \mathrm{~m}$ x $9000 \mathrm{~m}$ (voir la figure 13). Le domaine a été discrétisé en 26000 éléments. La cote des fonds varie de $-20 m$ (fond de la mer) à $100 m$ (fond du réservoir). Le barrage est modélisé par une ligne droite entre les points de coordonnées $(4701.18 \mathrm{~m}, 4143.41 \mathrm{~m})$ et $(4655.5 \mathrm{~m}, 4392.10 \mathrm{~m})$. La condition initiale consiste en une masse d'eau à la cote $100 \mathrm{~m}$ en amont du barrage. Le niveau de la mer est constant et égal à zéro. Excepté dans le réservoir et en mer, le fond est sec bien que la porte de sortie ait été ouverte. En amont du réservoir, nous avons imposé une constante du débit égale à zéro. Au temps $t=0 \mathrm{~s}$, il y a ainsi un mur d'eau de $55 \mathrm{~m}$ de haut à l'emplacement du barrage. L'évènement dure $4000 \mathrm{~s}$. Le calcul est fait avec un pas de temps de $0.05 \mathrm{~s}$.

Les données disponibles sont les temps d'arrivée de l'onde et la cote maximale de l'eau à un certain nombre de points d'observations et les mesures provenant d'un modèle physique à l'échelle $1 / 400$, réalisé par le Laboratoire National d'Hydraulique (LNH) de l'EDF en 1964 et calibré pour les données d'observations. Parmi 100 points d'observation, nous avons choisi ceux qui sont les plus significatifs pour la comparaison : A, B, C et S6-S14. Les temps de transit entre les transformateurs électriques A, B et C observés et calculés par (Valiani et al., 2002), par TELEMAC (Hervouet et Petitjean, 1999) et par notre modèle (baptisé ECOMOD-UFVM) sont donnés dans le tableau 1. Clairement, l'accord entre nos résultats calculés et les observations est très satisfaisante lorsque $C_{h}=25 \mathrm{~m}^{1 / 2} / \mathrm{s}$ et $A_{H}=15 \mathrm{~m}^{2} / \mathrm{s}$, surtout pour les points B et C. L'erreur relative sur le temps d'arrivée de l'onde (temps de coupure électrique) au point $\mathrm{A}$ obtenue par le présent modèle et par TELEMAC, est de $-15 \%$, et de $11 \%$, respectivement.

Le profil longitudinal de la surface libre maximale, passé par les points observés, représente une enveloppe du front de l'onde qui se propage pendant l'accident. La figure 14 compare ce profil donné par le modèle physique avec les résultats numériques obtenus par (Valiani et al., 1996 ; Hervouet, 2003) et par notre modèle. 
On note qu'aux points $\mathrm{S} 7$ et $\mathrm{S}$, les résultats numériques sont tous défaillants. L'écart entre les résultats numériques et ceux du modèle physique est maximal à ces points. Alcrudo et Gil, (1999) font la même remarque dans leurs résultats. Il semble qu'au point S6 (près du barrage), les résultats obtenus par (Valiani et al., 2002) et TELEMAC (Hervouet, 2000) sont plus près des valeurs mesurées que ceux obtenus par notre modèle. Cependant, aux points S10, S11 et S12, c'est-à-dire plus vers l'aval, cette situation est inversée : les résultats de notre modèle approchent mieux les mesures physiques que les deux précédents modèles.

Une étude de sensibilité montre que l'influence de $A_{H}$ est très faible sur les temps de transit entre les transformateurs $\mathrm{A}, \mathrm{B}$ et $\mathrm{C}$, tandis que l'influence du coefficient de frottement sur les cotes maximales de la surface d'eau reste faible.

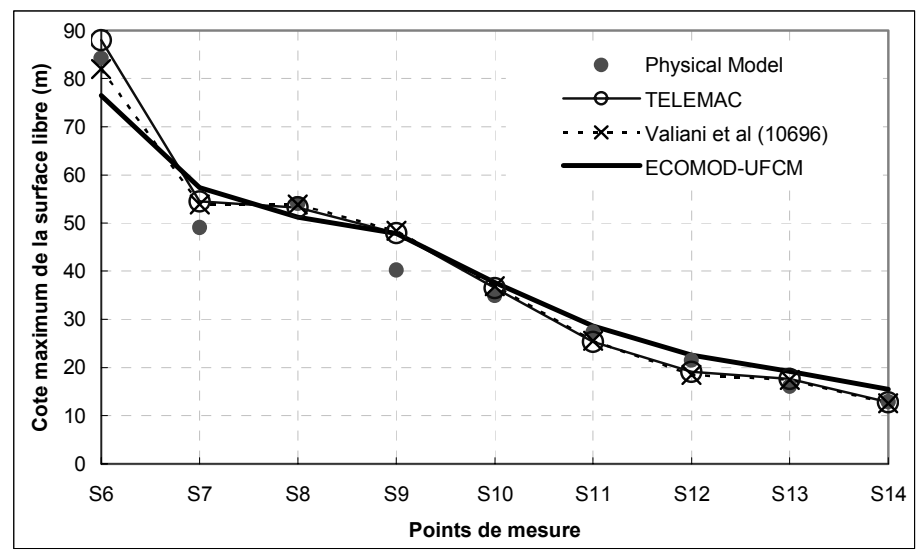

Figure 14. Cote maximale de la surface libre aux points de mesure sur le modèle physique comparé avec les résultats numériques

La figure 15 reproduit le champ de vitesses en aval du barrage à $100 \mathrm{~s}$ après la rupture. L'effet du virage est évident avec un début de recirculation vers la rive droite. La hauteur d'eau est représentée dans la figure 16 à l'instant $\mathrm{t}=4000 \mathrm{~s}$. La figure 17 présente le champ de vitesses à 4000 s après la rupture du barrage.

Cette simulation montre que le modèle est capable de reproduire avec précision un évènement réel, qui est la propagation des ondes dues à la rupture du barrage de Malpasset. Le traitement du terrain découvrant et recouvrant, qui est un problème délicat, a bien fonctionné. 
740 REMN - 16/2007. Modélisations numériques en mécanique

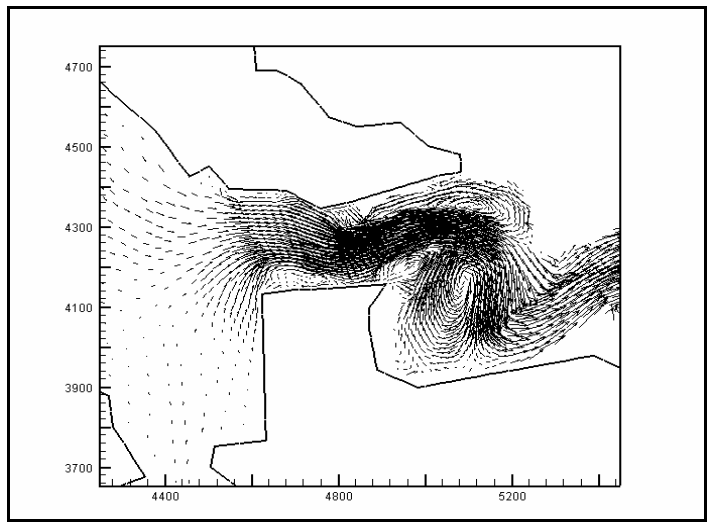

Figure 15. Champ de vitesses après $100 \mathrm{~s}$

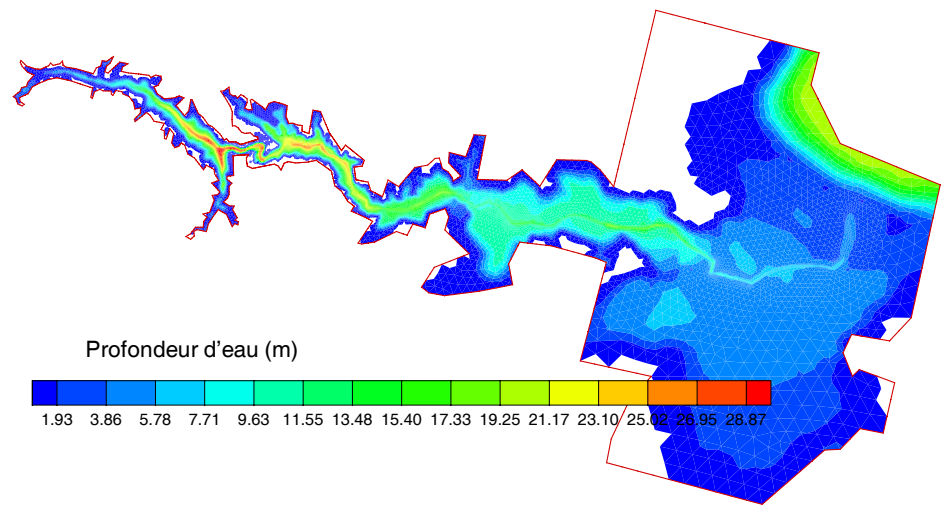

Figure 16. Profondeur d'eau à $t=4000 \mathrm{~s}$ après la rupture

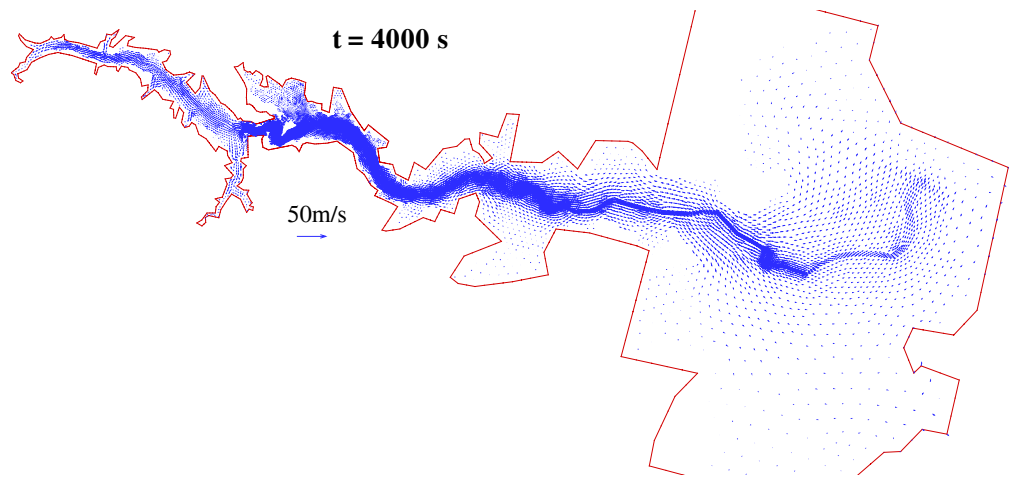

Figure 17. Champ de vitesses à $t=4000$ s après la rupture 


\subsection{Rupture de la digue dans le district de Thanh-Ha}

Le code 1-D TL (Nguyen et al., 2003), qui est crée par l'IMECH (Institut de Mécanique au Vietnam) est basé sur la résolution de l'équation de Saint-Venant unidimensionnelle. Notre modèle a été couplé au 1-D TL pour simuler l'écoulement dû à la rupture de la digue et leur propagation dans le district de Thanh-Ha pendant la crue de 1996 dans le delta du Fleuve Rouge (Nord du Vietnam).

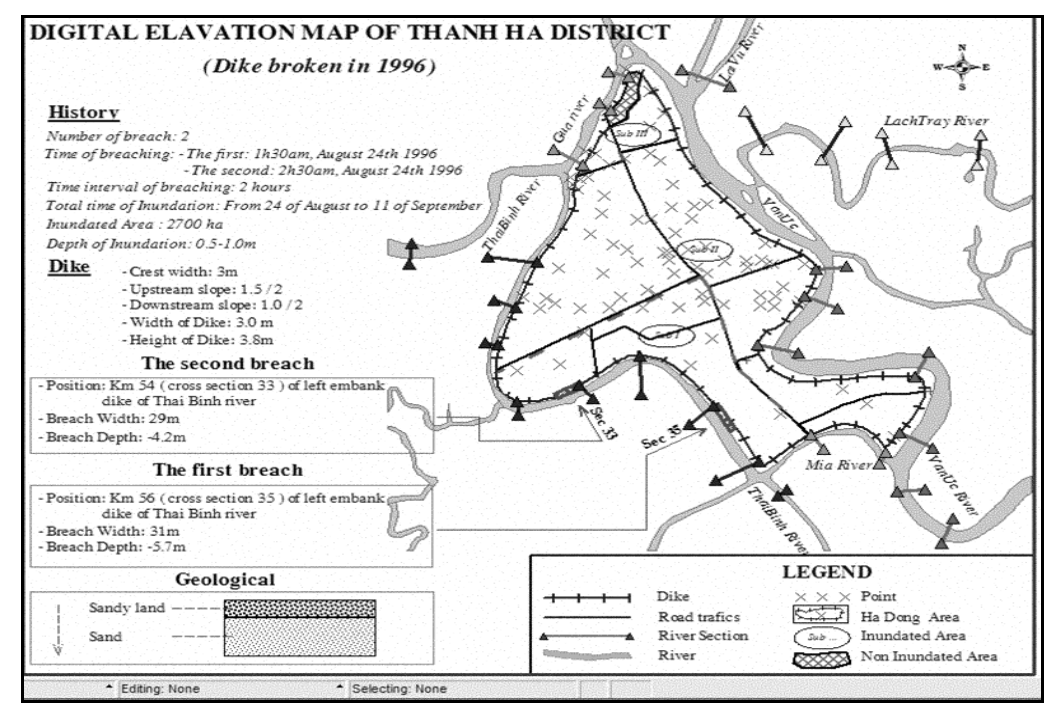

Figure 18. Carte géographique de Thanh-Ha

La figure 18 présente la carte géographique de la zone étudiée. Le district de Thanh Ha (Province de Thai-Binh, Vietnam du nord) est entouré par la rivière ThaiBinh à l'ouest, la rivière de Van-Uc à l'est, la rivière Gia et Mia au nord et au sud, respectivement. Ce district est protégé des crues de ces rivières par un système fermé de digues de $3.80 \mathrm{~m}$ de hauteur et $3.00 \mathrm{~m}$ de largeur. Au centre du district, une route départementale, qui traverse du sud-est au nord-ouest sur toute la longueur du district, le sépare en deux parties ouest et est (figure 18). Le 24 août 1996, deux segments de digue sur la rivière Thai-Binh à l'ouest du district étaient cassés : la première rupture a eu lieu à la section 33 à 1:30 du matin et la deuxième à la section 35 à 2:30 du matin. L'évolution des brèches de digue dure 2 h. 2,700 ha du district ont été inondés pendant 17 jours (du 24 août au 11 septembre) avec une profondeur variant de $0.5 \mathrm{~m}$ à $1.0 \mathrm{~m}$. Seule une zone de très faible superficie à l'extrême nord (indiquée en 'Non Inundated Area' dans la figure 18) du district a été épargnée de l'inondation. 


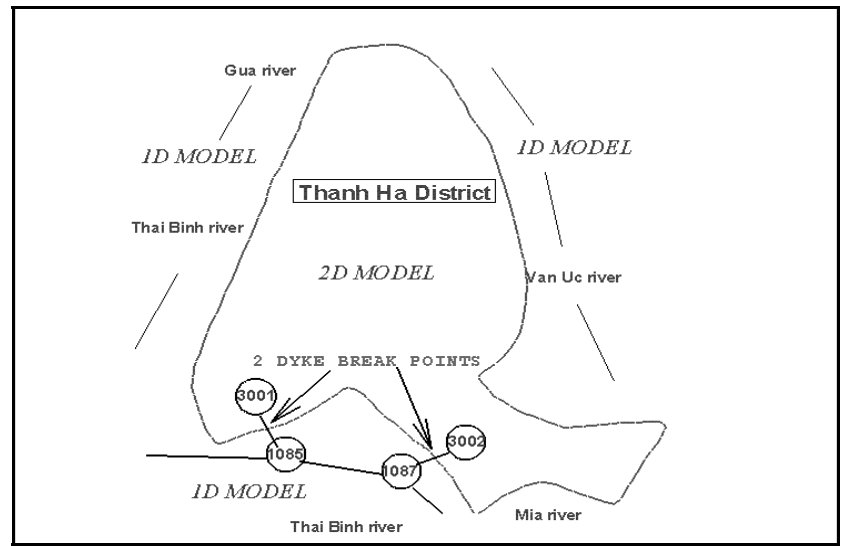

Figure 19. Schéma du couplage entre deux modèles

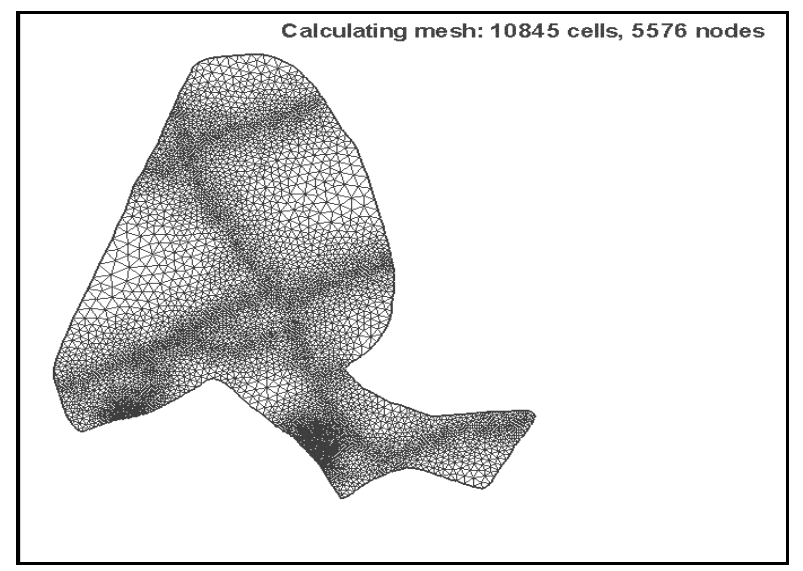

Figure 20. Maillage de la zone de Thanh-Ha

Le domaine a été discrétisé en 10845 triangles (figure 20). Notre modèle 2D a été couplé au modèle de 1-D TL par 2 noeuds (figure 19). Comme le pas de temps du modèle $2 \mathrm{D}$ est de $2 \mathrm{~s}$, alors qu'il est de $1 h$ pour le modèle 1 -D TL, nous devons calculer 1800 pas de temps du modèle 2D pour 1 pas de temps du 1-D TL. Le calcul est effectué durant $15 \mathrm{~h}$. La figure 21 présente les champs de vitesse et les contours de la profondeur d'eau à $\mathrm{t}=1,5,10$ et $15 \mathrm{~h}$. On voit nettement que jusqu'à $10 \mathrm{~h}$ après la rupture des digues, seule la partie ouest du district est effectivement inondée jusqu'à une hauteur de $1 \mathrm{~m}$ (figure $21 \mathrm{c}$ ). La route départementale a joué le rôle d'une digue secondaire permettant d'épargner la partie est de l'inondation. Au-delà de cet instant, l'eau commence à déverser sur la partie est. La profondeur y reste 
cependant faible (moins de $0.40 \mathrm{~m}$ ). La zone se trouvant au nord du district n'est pas inondée comme ce qui a été observé. La profondeur d'eau calculée dans les zones inondées, qui varie entre $0.40-1.00 \mathrm{~m}$, correspond aussi bien aux valeurs historiques observées.

Figure 22 montre la comparaison de la dénivellation de la surface d'eau obtenue par le couplage du code 1-D TL au modèle 2D et celle calculée par le code 1-D TL seul, sans le couplage, aux points de références. Une bonne accordance est obtenue.
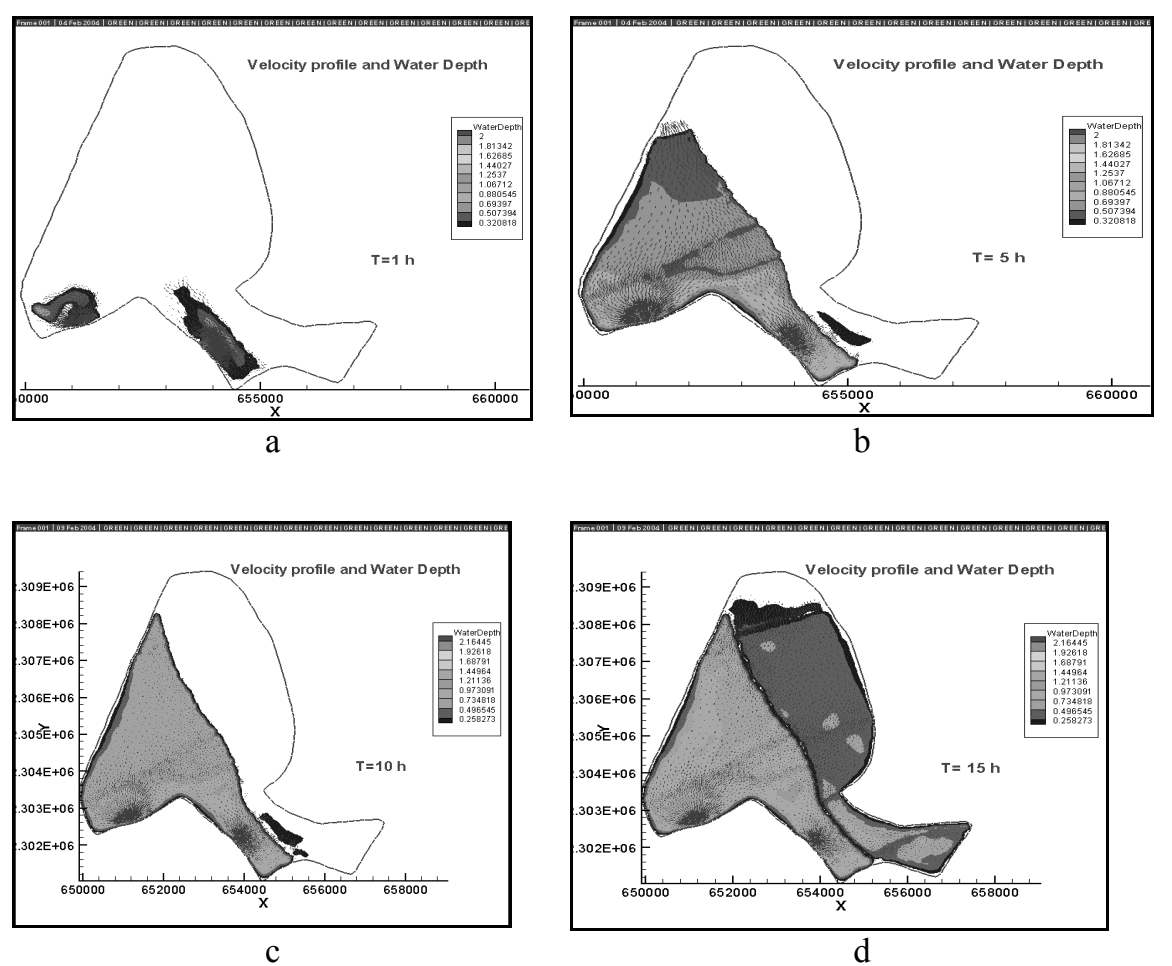

Figure 21. Champs de vitesse et contours de la profondeur d'eau a) $t=1 \mathrm{~h}$; b) $t=5$ $h$;c) $t=10 \mathrm{~h}$; d) $t=15$ 


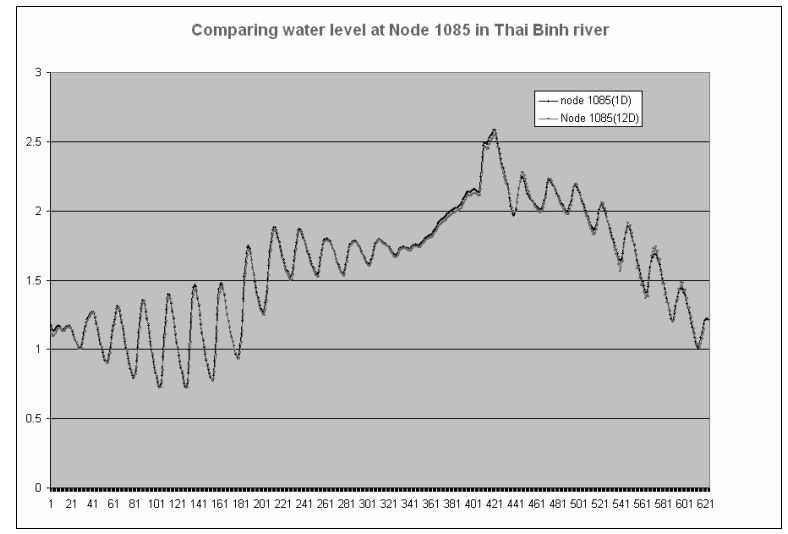

a

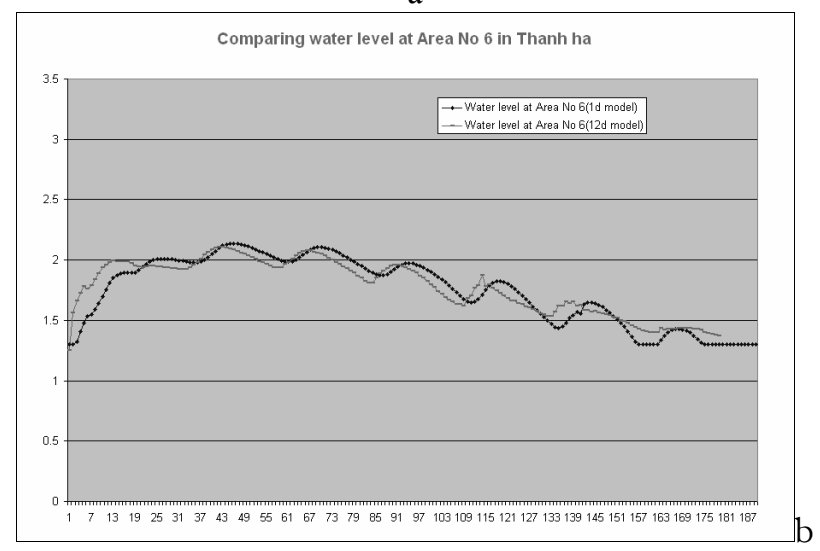

b

Figure 22. Comparaison de la dénivellation de la surface d'eau, a) obtenue par le couplage du 1-D TL au 2-D modèle ; b) celle obtenue par 1-D TL

\section{Conclusion}

Plusieurs cas-tests réalisés ont prouvé la capacité du modèle proposé à calculer des écoulements horizontaux sur des fonds irréguliers. L'application de l'onde due à la rupture du barrage de Malpasset prouve que ce modèle est capable de traiter les problèmes de terrains découvrants et recouvrants. L'application de ce modèle avec un couplage au modèle 1-D TL pour évaluer le delta du Fleuve Rouge est également réussie.

Le modèle basé sur la technique de projection utilisant une méthode des volumes finis non structurés est très approprié à la résolution des équations de Saint-Venant, même lorsque le terme source est important du à la variation rapide du fond. 


\section{Remerciements}

Le développement de ce modèle a eu un soutien financier de la commission européenne dans le cadre du projet FP5-FLOCODS ( ${ }^{\circ}$ ICA4-CT2001-10035).

\section{Bibliographie}

Alcrudo F., Garcia-Navarro P., "A high-resolution Godunov-type scheme in finite volumes for the 2D shallow-water equations", Int. J. Numer. Meth. Fluids, vol. 16, n 6, 1993, p. $489-505$.

Alcrudo F., Gil E., "The malpasset dam break case study", The Proceeding of the $4^{\text {th }}$ CADAM meeting, Zaragoza, Spain, 1999. p. 95-109.

Borthwick A.G.L., Karr E.T., "Shallow flow modeling using curvilinear depth-averaged stream function and vorticity transport equations", Int. J. Numer. Meth. Fluids, vol. 17, p. 417-445.

Brufau P., Garcia-Navarro P., Vázquez-Cendón M.E., "Zero mass error using unsteady wetting-drying conditions in shallow flows over dry irregular topography", Int. J. Numer. Meth. Fluids, vol. 45, 2004, p. 1047-1082.

Brufau P., Vázquez-Cendón M.E., García-Navarro P., "A numerical model for the flooding and drying of irregular domains", Int. J. Numer. Meth. Fluids, vol. 39, 2002, p. 247-275.

Caleffi V., Valiani A., Bernini A., "Fourth-rder balanced source term treatment in central WENO schemes for shallow water equations", J. Comp. Phys, vol. 218, 2006, p. 228-245.

Chan C.T., Anastasiou K., "Solution of incompressible flows with or without a free surface using the finite volume method on unstructured triangular meshes", Int. J. Numer. Meth. Fluids, vol. 29, 1999, p. 35-57.

Chorin A. J., "Numerical solution of the Navier-, n 1, 2000, p. 35-58, "Stokes equations". Math. Comput., vol. 22, $\mathrm{n}^{\circ}$ 104, 1968, p. 745-762.

Chorin A.J., "On the convergence of discrete approximations to the Navier-Stokes equations" Math. Comput., vol. 23, 1969, p. 341-353.

Fennema R., Chaudry M., "Explicit methods for 2-D transient free-surface flows", J. Hydraul. Eng., ASCE, vol. 116, n 8, 1990, p. 1013-1034.

Garcia-Navarro P., Alcrudo F., "Saviron J.M. 1-D open-channel flow simulation using TVDMcCormack scheme", J. Hydraul. Eng., ASCE, vol. 118, n 10, 1992, p. 1359-1372.

Guillou S., Modélisation des écoulement côtiers et estuariens : étude mathématique et applications en coordonnes généralisées, Thèse de doctorat, Université Paris VI, 1996.

Guillou S., Nguyen K.D., "An improved technique for solving two-dimensional shallow water problems”, Int. J. Numer. Meth. Fluids, vol. 29, n 4, 1999, p. 465-483.

Hanich L., Résolution des équations de la mécanique des fluides par des méthodes TVD en coordonnées généralisées, Thèse doctorale, Université de Caen, 1996. 
Harten A., Engquist B., Osher S., "Chakravarthy S.R. Uniformly high order accurate nonoscillatory schemes”, J. Comp. Phys, vol. 71, 1987, p. 231.

Hervouet J.M., Petitjean A., "Malpasset dam break revisited with two-dimensional computations", Journal of Hydraulic Research, vol. 37, n 6, 1999, p. 777-788.

Hervouet J.M., “A high resolution 2-D dam-break model using parallelization”, Hydrological Processes, vol. 14, $\mathrm{n}^{\circ} 13,2000$, p. 2211-2230.

Hervouet J.M., Hydrodynamique des écoulements à surface libre: Modélisation numérique avec la méthode des éléments finis, Paris, Presses de l'Ecole Nationale des Ponts et Chaussées, 2003.

Kobayashi M.H., Pereira J.M.C., Pereira J.C.F., "A conservative finite-volume second-order accurate projection method on hybrid unstructured grids", J. Comp. Phys, vol. 150, n 1 , 1999, p. 40-75.

Lien F.S., "A pressure-based unstructured grid method for all-speed flows", Int. J. Numer. Meth. Fluids, vol. 33, n 3, 2000, p. 355-374.

Murillo J., Garcia-Navarro P., Brufau P., Burguete J., "Extension of an explicit finite volume method to large time steps (CFL $>1)$, application to shallow water flows", Int. J. Numer. Meth. Fluids, vol. 50, 2005, p. 63-102.

Nguyen V.H., Nguyen V.D., Ngo, H. C., "On some numerical methods for solving the 1-D Saint-Venant Equations of general flow regime, Part 2: Verification and Application", Vietnam Journal of Mechanics, NCST de Vietnam, vol. 25, $\mathrm{n}^{\circ}$ 1, 2003, p. 26-38.

Rhie C.M., Chow W.L, "Numerical study of the turbulent flow past an airfoil with trailing edge separation", AIAA J., vol. 21, n 11, 1983, p. 1525-1532.

Roe P.L., "Approximate Riemann solvers, parameter vectors and difference schemes", J. Comp. Phys., vol. 43, n 2, 1981, p. 357-372.

Ryhming I.L., Dynamique des fluides, Presses Polytechniques et Universitaires Romandes, 1985.

Saint-Venant, A.J.C., Théorie du mouvement non-permanent des eaux, avec application aux crues des rivières et à l'introduction des marées dans leur lit, Compte-Rendu à l'Académie des Sciences de Paris, vol. 73, 1871, p. 147-154.

Sleigh P.A, Gaskell P.H., Berzins M., Wright N.G., "An unstructured finite-volume algorithm for predicting flow in rivers and estuaries”, Comput. Fluids, vol. 27, n 4, 1998, p. 479-508.

Stevens D.E, Chan S. T., Gresho P., "An approximate projection method for incompressible flow”, Int. J. Numer. Meth. Fluids, vol. 40, 2002, p. 1303-1325.

Tan W., Shallow water hydrodynamics, Amstrerdam, The Netherlands, Elsevier, 1992.

Tannehill J.C., Anderson D.A., Pletcher R.H., Computational Fluid Mechanics and Heat Transfer, Chapter IV "Application of Numerical Methods to Selected Model Equations", $2^{\text {nd }}$ Edition, Taylor \& Francis, Washington D.C., p. 156-158, 1997.

Temam R., "Une méthode d'approximation de la solution des équations Navier-Stokes", Bull. Soc. Math. France, vol. 98, n ${ }^{4}$, 1968, p. 115-152. 
Temam R., Navier-Stokes Equations, North-Holland, Amsterdam, 1977.

Valiani A., Caleffi V., Zanni A., "Case study: Malpasset dam-break simulation using a twodimensional finite volume method", J. Hydraul. Eng., ASCE, vol. 128, n 5, 2002, p. 460472.

Van Leer B., "Towards the ultimate conservative difference scheme, IV, A new approach to numerical convection", J. Comp. Phys, vol. 23, n 3, 1977, p. 276-299.

Vukovic S, Sopta L., "ENO and WENO schemes with the exact conservation property for one-dimensional shallow water equations", J. Comp. Phys, vol. 179, n 2, 2002, p. 593621.

Zhao D.H., Shen H.W., Tabious G.Q., Lai J.S., Tan W.Y., "Finite-volume two-dimensional unsteady-flow model for river basin", J. Hydraul. Eng., ASCE, vol. 120, n 7, 1994, p. $864-883$.

Zhou J.G., Causon D.M., Mingham C.G., Ingram D.M., "The surface gradient method for the treatment of source terms in the shallow-water equations", J. Comp. Phys, vol. 168, n 1, 2001, p. 1-25. 
